\title{
Mental Development Index
}

National Cancer Institute

\section{Source}

National Cancer Institute. Mental Development Index. NCI Thesaurus. Code C81263.

A sequential arrangement of information pertaining to the evolution of an individual's cognitive development. 